\title{
Heart Rate, Stroke Volume and Vasomotor Regulation in the Cat during Exertion and Postural Changes*
}

\author{
Giuseppe Mancia, Giorgio Baccelit, David B. Adams ** and Alberto Zanchetti \\ Cardiovascular Research Institute, University of Milan, and Center of Cardiovascular Research, \\ National Research Council, Milan, Italy
}

Received: May 25, 1970, and in revised form: Juli 17, 1970

\begin{abstract}
The cat, when its peripheral metabolic demands are augmented, has a limited capacity to increase its heart rate, but often responds with a considerable rise in stroke volume: stroke volume can be increased by active changes of posture from lying to standing, by a short or prolonged bout of fighting, and by mild exercise on the treadmill. In all these conditions stroke volume attains values larger than those measured during recumbent rest. It appears therefore that an increase in stroke volume is a mechanism ordinarily called upon in the cat when natural behaviour requires an increase in cardiac output, while
\end{abstract}

in man it is called upon in extraordinary conditions only. However, the fact that a stroke volume regulating mechanism is ordinarily operative in the cat shows that it has a physiological significance, and supports the idea that in man also exertion is accompanied by a masked stimulation of myocardial contractility. A description is also presented of the vasomotor changes produced by active changes in posture.

Key-words: Heart rate, stroke volume, exercise, posture, vasomotor changes, mesenteric circulation, iliac circulation, sympathetic cardiac regulation, vagal cardiac regulation.
The mechanisms by which heart rate and stroke volume are regulated in man during exertion and postural changes are still somewhat controversial. According to most authors stroke volume at rest is maximal in the supine position, and considerably decreases upon assumption of the sitting or standing postures; during exercise in any posture, tachycardia is the main response, and stroke volume would augment above the reduced standing resting values, but not significantly above supine resting values, which would be the maximal attainable levels of stroke volume in any condition (Rushmer, 1959; Bevegård et al., 1960; Wang et al., $1960 \mathrm{~b}$; Damato et al., 1966; Bevegård and Shepherd, 1967).

This conception has been partly challenged by Chapman et al. (1960), who have shown that during extremely severe exercise, when the beart rate has reached its top values and cannot rise farther, an additional increase in cardiac output is obtained through an increment in stroke volume, which is made considerably greater than in supine rest. That cardiac output during exertion can rise through an increase in stroke volume, rather than in heart rate, is also shown by experiments in which the heart rate was maintained fixed by atrial pacing (Ross et al., 1965) or was partly controlled by cholinergic blockade (Bevegård, 1963). However, these experiments do not indicate how often and to what extent the stroke volume regulating mechanism is operative in more natural conditions when the heart rate is free to increase; nor do they answer the question whether the stroke volume can really and

* Presented in part at the Fire-Side Chat on Heart Rate Regulation, during the 4 th Annual Meeting of the European Society for Clinical Investigation, Scheveningen, 1970.

* Post doctoral Fellow of the Public Health Service from Yale University, New Haven, Conn. (U.S.A.). significantly increase above base-line values during supine rest at a spontaneous low heart rate.

We have thought that, if stroke volume regulation is a basic natural mechanism through which the heart can fulfil peripheral metabolic demands, this mechanism might be more easily observed and studied in a species, such as the cat, in which the heart rate is naturally regulated, so to speak, at the opposite end of the frequency range as compared to man. In man, because of predominance of tonic vagal influences (Robinson et al., 1966) the base-line heart rate is generally set close to the lower end of its range, having a very limited capacity to decrease in a behaviour such as sleep (Bristow et al., 1969), but being able to increase more than threefold during exertion. On the other hand, a very low resting vagal tone (Guazzi et al., 1968) makes the cat's heart rate very high in base-line conditions, generally over 160 beats/min and often above 200 . Consequently, the cat's heart has a wide ability to decrease its rate during sleep (Guazzi and Zanchetti, 1965; Guazzi et al., 1968) but a very limited one to increase it during exertion (Adams et al., 1969 and 1970).

The experiments of which the results are being reported below have been aimed at clarifying cardiac regulation during active postural changes and during exertional tasks in both the reclining and standing postures. It will be seen that the cat seems to be naturally possessed of very effective mechanisms for regulating stroke volume during ordinary behaviour, an observation which suggests that these mechanisms have a role in circulatory adjustment in man. We have also taken advantage of this study for obtaining in the cat a more complete description than the one available in man of the vasomotor adjustments occurring during active postural changes. 
Methods

The data reported in this paper come from five cats ( $\mathrm{G}, \mathrm{R}, \mathrm{O}, \mathrm{B}, \mathrm{M})$, which were studied in the unanaesthetized unrestrained state for a total amount of 112 trials, as detailed below.

Three natural behaviours have been investigated while the cats were kept in a large cage with transparent walls: spontaneous assumption of the standing position, in order to record the circulatory effects of active postural changes; and two exertional tasks, one being fighting against another cat either in the lying or standing posture, the other being a mild standing exercise on a treadmill.

Cats $R, G, O$ and $M$ were observed while they spontaneously changed, from time to time, their body position from lying on one side (henceforth called lying) to quiet standing on four feet (henceforth called standing). Six episodes of postural change were analysed in cats $G, R$ and $O$, and four episodes in cat $M$.

Fighting was elicited in all five cats as a natural reaction to an attack brought forth by another cat, in which the attack was induced by brain stimulation (Adams et al., 1969). Both the attacking and the reacting cats were in the same cage, but separated by an opaque screen, which was raised whenever a fighting trial had to be provoked. Natural fighting was studied both when the reacting cat was lying and standing. In one position, the animal lay on its side, hissed, and struck with the forelimbs during fighting, but did not raise itself on the hindlimbs or use them in the fighting. In the other condition, the animal supported itself on the four limbs during the base-line period, in either the sitting or standing position, and then used the hindlimbs for balance and support during the fighting. Six episodes of fighting in the lying and six in the standing posture were studied in each of the five cats, each period of fighting being kept short to 2-10 sec. In cats $\mathrm{G}$ and $\mathrm{O}$ six trials of more prolonged fighting, lasting $20-30 \mathrm{sec}$ were also performed. Other types of fighting responses, those in which the cat rose from lying to standing during fighting, or in which the cat rolled over onto its side changing from standing to lying during the fighting, were not analysed.

For the study of a mild standing exercise cats $R$, $G$ and $O$ were observed while walking on a treadmill at the slow rate of 10 metres per minute. The cat was standing before the trial was begun, and an episode was initiated when the treadmill was turned on and the cat immediately began to walk. No emotional reaction was apparent, as the cats had been given prolonged training and the exercise was a very mild one. Six episodes were studied in each of the three cats.

The following cardiovascular measurements were continuously made in all cats throughout all kinds of trials. An electromagnetic flow probe (Statham $K$ probe of the hinged type, 6-7 $\mathrm{mm}$ i.d.) was implanted around the ascending aorta, and used to measure either cardiac output or stroke volume (less coronary flow) and to trigger a cardiotachographic device for automatic display of heart rate. Two other electromagnetic flow probes (Statham Q probes of the slot type, 1.5-2.0 mm i.d.) were implanted around the superior mesenteric artery and the left external iliac artery. All probes were connected to three Statham M - 4001 modules and power supply. The sensitivity of the flowmeters had been checked by calibration with blood and with saline before implantation in the cats, and was rechecked at the end of chronic recording with results that agreed within $10 \%$.Zero-flow level was the diastolic level in the case of the aorta, whereas for the two peripheral arteries it was repeatedly established by occlusion of the descending aorta by transient pulling of a snare implanted well above the origin of the superior mesenteric artery.

The three flow probes as well as the aortic snare were aseptically implanted under pentobarbital anaesthesia, following left thoracotomy and a wide midline opening of the abdomen. The animals usually recovered in less than 1 week during which time they were maintained in a thermostatic cage and under antibiotic treatment. Cables from the flow probes and one end of the snare were passed through the paravertebral muscles and the overlying skin, and protected in a leather packet sewn to the skin of the back.

One or two days before the recording sessions, under transient ether anaesthesia, a polyethylene tubing with a sufficiently rigid wall to prevent damping of the pressure pulse was inserted into the right femoral artery, and its free end also passed into the back packet. From this time on, the animal was heparinized. For the recording of blood pressure the arterial cannula was connected with a Statham strain-gauge transducer feeding into the bridge circuit of a d.c. preamplifier of a 12-channel Grass P7 inkwriter. On three channels of the same polygraph were displayed the instantaneous flow signals of the three flowmeters. Cardiac output (less coronary blood flow), mesenteric blood flow and blood flow through the left hindlimb were continuously computed from the instantaneous flow curves by integrating amplifiers (Grass 7 P 10), automatically reset at 2 -sec intervals, and were displayed on three other channels of the polygraph. The heart rate appeared on a further channel, calculated beat by beat by a cardiotachograph triggered by the aortic flow pulse.

Two-second measures of cardiac output, mesenteric and iliac flow, and heart rate could be taken directly from integrator and cardiotachographic tracings. Stroke volume was calculated from 2-sec measures of cardiac output and heart rate, or measured beat by beat as the deflections of the aortic flow integrator automatically reset at each diastolic interval. Mean arterial pressure was computed for the same interval of 2 sec as systolic plus twice diastolic pressure divided by three. Total peripheral, mesenteric and iliac conductances were computed for each 2-sec interval by dividing each flow by mean arterial pressure. Reasons favouring the use of 


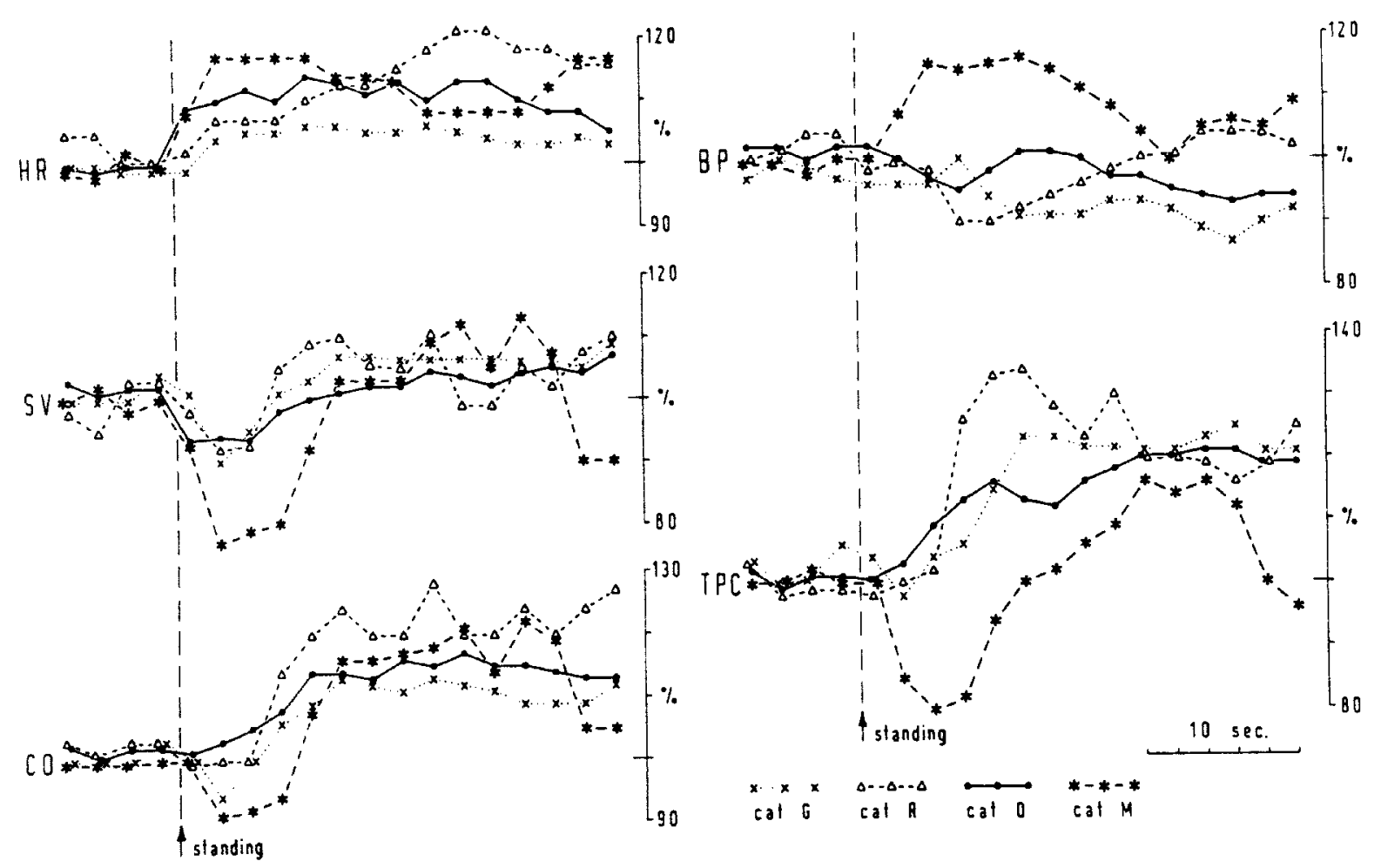

Fig. 1. Changes in heart rate (HR), stroke volume (SV), cardiac output (CO), mean blood pressure (BP) and total peripheral conductance (TPC) during spontaneous assumption of the standing posture (change in posture indicated by vertical broken line) in four cats (each identified by a different symbol, as indicated in the figure). Each symbol is mean of six 2 -sec measurements (except for cat M: four measurements only). Values are expressed as a percentage of base-line values randomly chosen in the 30 sec preceding the change in posture

conductance rather than resistance have been pointed out elsewhere (Stark, 1968; Adams et al., 1969).

The 2 -sec measurements of the different cardiovascular variables were analysed for the 8 sec preceding and the 30 sec following the appearance of electromyographic activity in the left hindlimb, signalling the spontaneous change of the cat from the lying to the standing posture. In the fighting trials, analysis was done of 2 -sec measurements for $6 \mathrm{sec}$ before opening the partition separating the attacking from the reacting cat, and for $30 \mathrm{sec}$ following the first striking movement signalling the fighting response of the reacting cat. In exercise trials, similar analysis was carried out for $6 \mathrm{sec}$ before and $20 \mathrm{sec}$ after the cat started walking on the treadmill. Base-line values were obtained from 2-sec intervals randomly selected from a 30 -sec period immediately prior to each trial. Values recorded in the different postures were compared, when deemed necessary, by analysis of variance with two way classification (Snedecor and Cochran, 1967).

\section{Results}

Heart Rate, Stroke Volume and Vasomotor Responses to Spontaneous Changes in Body Posture. Fig. 1 summarizes haemodynamic changes measured in four cats when they spontaneously and quietly turned from lying on one side to standing on the four feet. On changing posture, the heart rate promptly rose. This increase, though statistically significant in all cats $(p<0.01$ or $<0.05$ ), was however rather limited, amounting to $5-17 \%$ of control values. Significant tachycardia was still present in three cats toward the end of the 30-sec recording period. The stroke volume showed a prompt, short-lasting decrease to mean values of $77-93 \%$ of lying levels in the different animals. In all cats it was statistically significant $(p<0.05)$. However, after 8-12 sec from the change in posture stroke volume had at least resumed the base-line values in spite of persisting tachycardia. As a matter of fact, in all cats stroke volume somewhat increased above control values (means from 105-114\%), the increase being statistically significant in three cats $(p<0.01$ or $<0.05)$. Cardiac output was briefly and slightly reduced in two cats though for a few seconds only, and then significantly augmented in all cats (up to means of $113-127 \%$ of lying values) as the result of rises in both heart rate and stroke volume $(p<0.01)$.

Mean blood pressure was variously affected in the four cats. In three of them it was slightly reduced (to mean values of $87-93 \%$ of control), but the decrease was significant in two cats only. In one of these cats (R) the blood pressure fall was prompt but did not last 


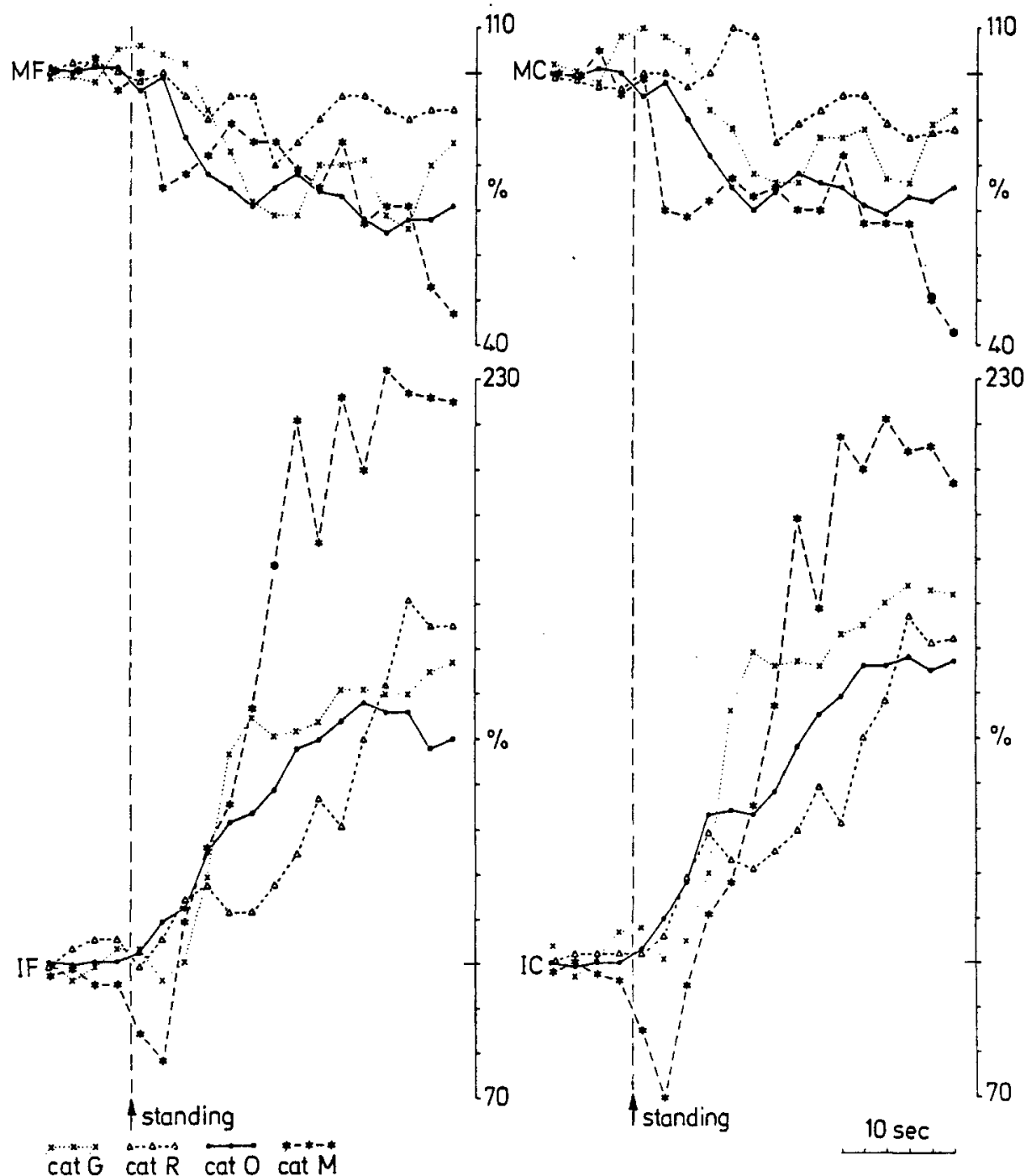

Fig. 2. Changes in superior mesenteric blood flow (MF) and conductance (MC), and in left external iliac blood flow (IF (and conductance (IC) during spontaneous assumption of the standing posture (change in posture indicated by vertical broken line). All other explanations as in Fig. 1

more than a few seconds, while in the other two cats there was a slight early decrease, followed by a short return to control values and then by a more marked fall. In the fourth cat (M), instead of a decrease, there was a significant increase in blood pressure up to $116 \%$ of control values. In cats $R, G$ and $O$, parallel to the decrease in blood pressure, there was a large significant increase in total peripheral conductance (120-138\% of lying values, $p<0.01)$, signalling an overall vasodilatation. In cat $M$ there was an initial significant vasoconstriction ( $80 \%$ of control total peripheral conductance, $p<0.01$ ) simultaneous to the decreased cardiac output, which finally gave way to the usual significant vasodilatation ( $116 \%$ of control total peripheral conductance, $p<0.01)$.

Fig. 2 shows that the overall vasodilatation seen in all cats upon standing was not a uniformly diffuse vasomotor reaction. In the external iliac bed there was a prompt and progressive increase in blood flow and a huge vasodilatation, which in cat $\mathrm{M}$ only was preceded by a short-lasting but significant vasoconstriction. External iliac conductance increased significantly in all cats, to values averaging $160-220 \%$ of control $(p<0.01)$. Simultaneously, however, decreased blood flow and vasoconstriction were observed in the superior mesenteric bed. Mesenteric conductance decreased significantly in all cats, to values averaging $67-85 \%$ of control $(p<0.01$ or $<0.05)$. It appears therefore that, on active assumption of the standing posture, vasodila. tation, presumably metabolic in origin, occurring in contracting muscles is partly compensated by visceral vasoconstriction.

Actual tracings from cat $O$ during spontaneous changes in posture are reproduced in Fig. 3. 


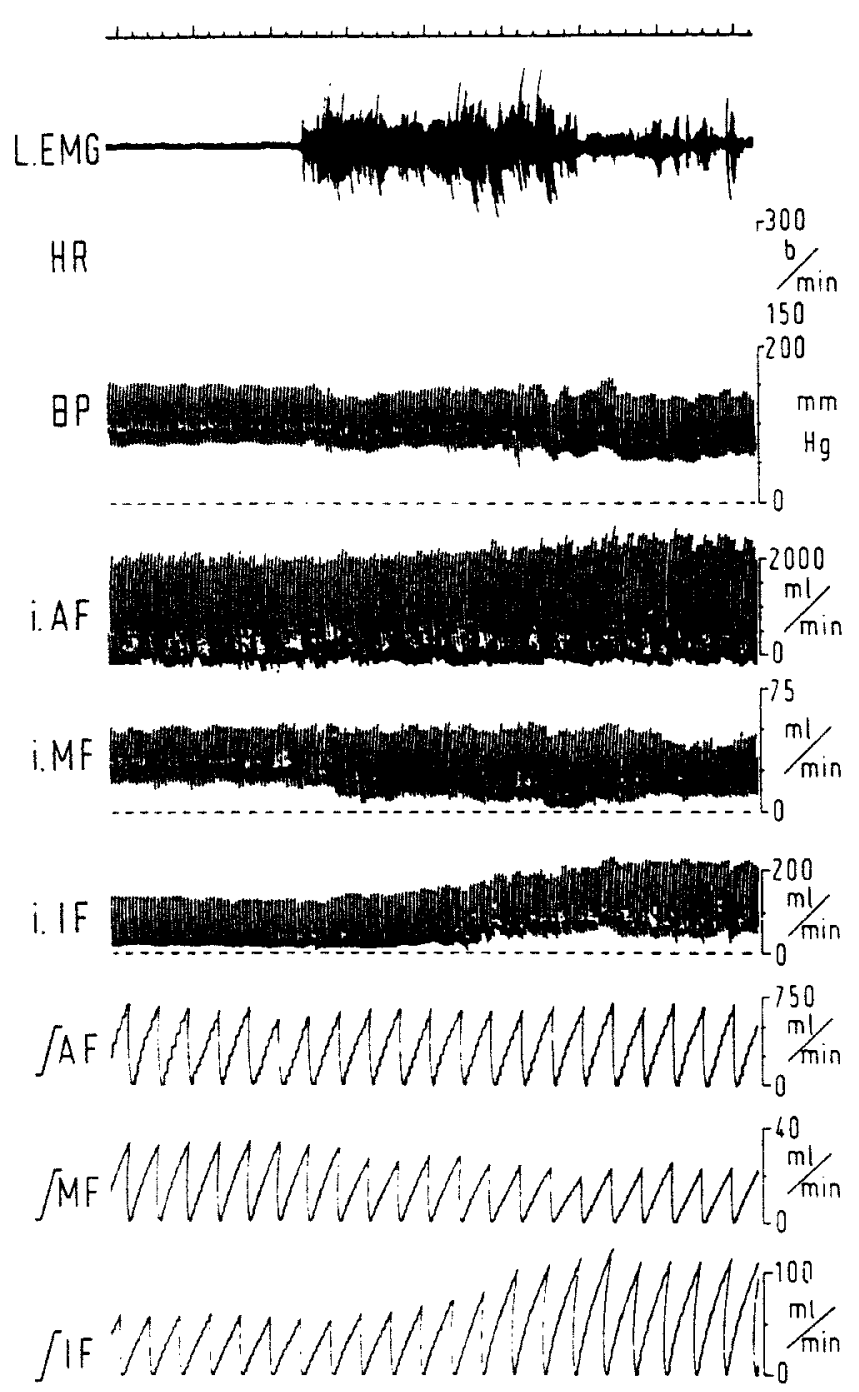

Fig. 3. Cardiovascular changes in cat $O$ during spontaneous assumption of the standing posture (change in posture indicated by arrow). From above downwards: time ( 1 and $5 \mathrm{sec}$ ); $L . E . I G$ electromyogram of the left hindlimb; $H R$ heart rate; $B P$ arterial blood pressure; $i A F$ instantaneous flow through the ascending aorta; $i M F$ instantaneous flow through the superior mesenteric artery; $i I F$ instantaneous flow through the left external iliac artery; $\int A F, \int M F, \int I F 2$-sec integrations of the above flows

Heart Rate and Stroke Volume Regulation during Exertion. Effects of Changes in Body Posture. Fig. 4 summarizes the changes in heart rate measured in 5 cats during an exertional task, a short period (2-10 sec) of fighting, performed both in the lying and the standing posture. With the single exception of cat $R$, the base-line heart rate before fighting was almost the same both in the standing and in the lying position. It rose during fighting, particularly during the very first seconds, but the increase was on the whole rather moderate, ranging from $10-40 \%$ above baseline values. Again with the exception of the cat $R$, tachycardia was somewhat greater when fighting occurred in the standing as

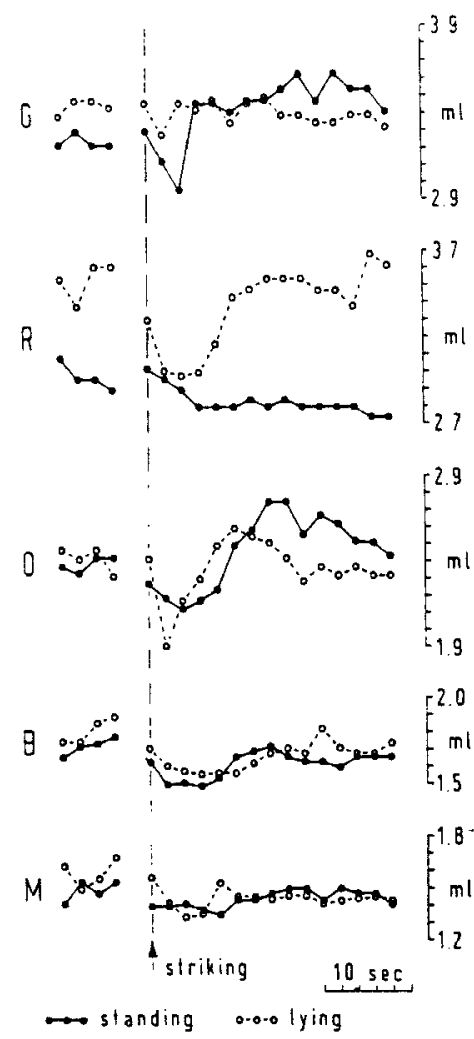

Fig. 4. Heart rate during fighting in the lying (empty circles, broken lines) or the standing position (filled circles, continuous lines). Each symbol is mean of six 2-sec measurements in different trials. Five cats indicated on the left by their initial

compared to the lying posture, but differences were generally not very prominent.

The changes in stroke volume measured in the same cats during fighting in the two positions are summarized in Fig. 5. Except for cat $R$, resting stroke volume was remarkably similar in the standing and the lying posture. During fighting in the lying position stroke volume initially decreased (markedly in cats $R$ and $O$ because of the conspicuous tachycardia, slightly in the other three animals), and then rapidly returned to resting levels. During a short flurry of fighting in the standing posture, the initial decrease in stroke volume (generally smaller than in lying trials) was followed in four of the five cats (cat $R$ being, as usual, the exception) by a rise in stroke volume to values which were larger than those measured during recumbent fighting, and in cats $G$ and $O$ to stroke volumes larger than those recorded in the same cats during recumbent rest (cat G: $3.63 \pm 0.08 \mathrm{ml}$ (mean \pm S.E.) during fighting on standing as compared to $3.49 \pm 0.09 \mathrm{ml}$ resting recumbent value; cat $O: 2.75 \pm 0.09 \mathrm{ml}$ as compared to $2.42 \pm 0.08 \mathrm{ml}$ ).

Greater percentage increases of stroke volume above lying resting levels (LR) could be observed in these two cats during more prolonged exertional tasks than a short bout of fighting (SF), as shown in Fig. 6 . 


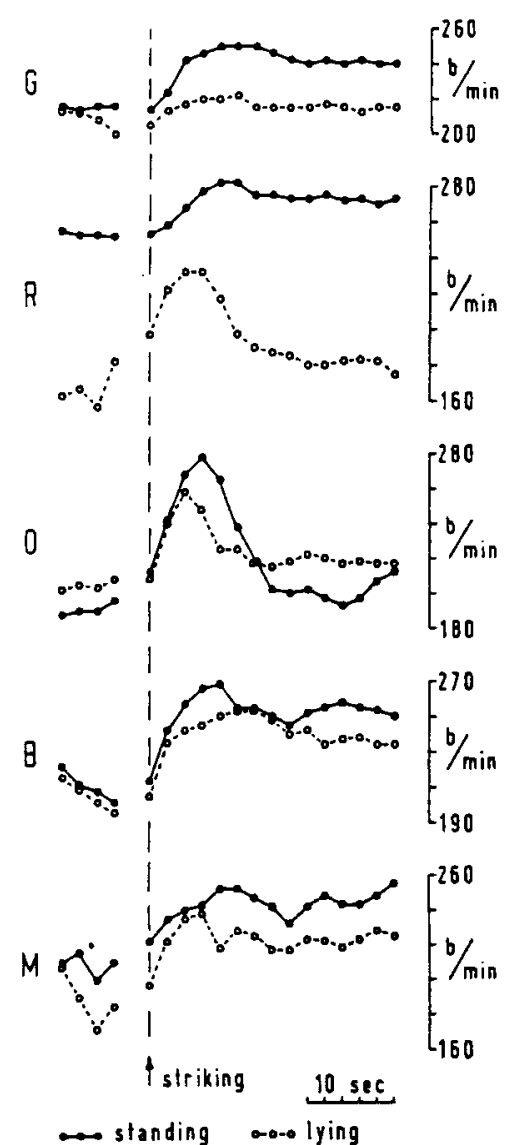

Fig. 5. Stroke volume during fighting in the lying or the standing position. Symbols as in Fig. 4

When fighting trials were prolonged for at least 20-30 sec (LF), cardiac output continued to increase to levels well above those occurring during shorter episodes, while the heart rate did not increase beyond the values attained at the beginning of fighting. As a consequence, long fighting caused conspicuous increases in stroke volume, which became larger than values recorded at lying rest. In cat $G$ the mean of the highest stroke volume at the end of six episodes of long fighting was $4.25 \pm 0.10$ as compared to $3.49 \pm 0.09 \mathrm{ml}$, the mean of six resting recumbent values (an increase of $22 \%$ ); in cat $O$ the corresponding means were $3.12 \pm 0.09$ and $2.42 \pm 0.08$ (an increase of $29 \%$ ).

As also shown in Fig. 6, stroke volume was measured in these two cats during a mild standing exercise, namely walking on a treadmill at low speed: in both cats the heart rate rose very slightly, and the rise in cardiac output was effected almost exclusively through an increase in stroke volume that became definitely larger during standing exercise $(\mathrm{E})$ than during a preceding period of rest in the recumbent position (LR) (cat G: $4.17 \pm 0.09 \mathrm{ml}$ as compared to $3.49 \pm 0.09 \mathrm{ml}$; cat $0: 2.75 \pm 0.07 \mathrm{ml}$ as compared to $2.42 \pm 0.08 \mathrm{ml}$ ).

Increases in stroke volume over the resting recum. bent values were statistically significant at the $p<0.01$

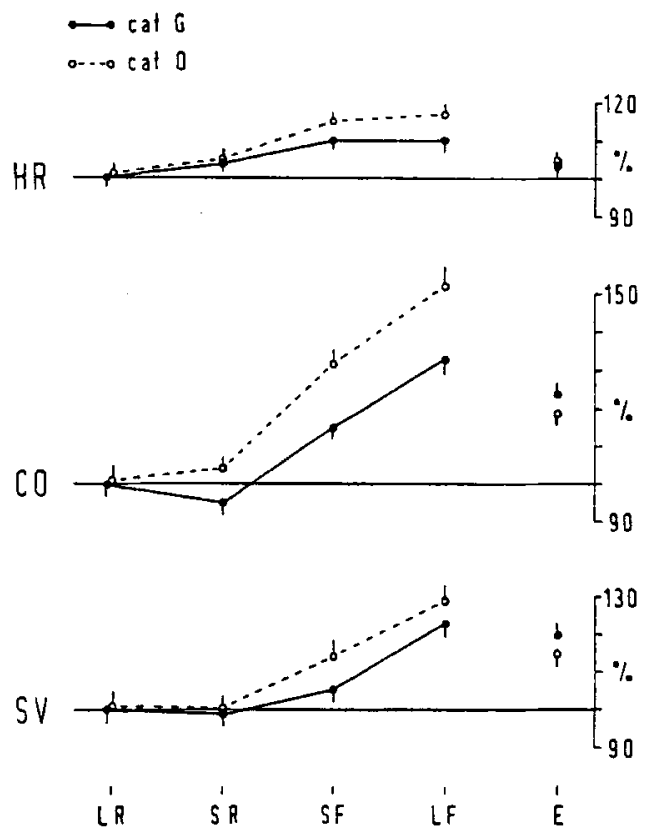

Fig. 6. Percentage changes in heart rate (HR), cardiac output (CO) and stroke volume (SV) in cats $\mathrm{G}$ (filled circles, continuous lines) and $O$ (empty circles, broken lines), during standing at rest (SR), short episodes of fighting (SP), long episodes of fighting (LF), and exercise on tredmill (E). LR: baseline values during lying at rest. Each symbol is mean of six 2-sec measurements in different trials. Bars indicate standard errors of the mean, and have been drawn on one side only of the symbols of the means to avoid overlapping between bars of either cat

level in both cats during short fighting and exercise. It should be clear from the data presented above as well as from Fig. 5 that the stroke volume increments observed in cats $G$ and $O$ were by no means an exception, but simply represented the upper end of a trend also evident in cats $B$ and $M$. Nor did cats $G$ and $O$ mark. edly differ from the other animals of the group in any of the haemodynamic functions measured, their baseline heart rate ( 215 and 210 beats/min), stroke index (19.28 and $13.08 \mathrm{ml} / \mathrm{m}^{2}$ ) and cardiac index (4.14 and $2.75 \mathrm{l} / \mathrm{min} / \mathrm{m}^{2}$ ) being well within the normal range for cats, and similar to the values measured in the other three cats.

\section{Discussion}

Our data show that the cat, when its peripheral metabolic demands are augmented, has a limited capacity to increase its heart rate, but often responds with a considerable rise in stroke volume: stroke volume can be increased by active changes of posture from lying to standing, by a short or prolonged bout of fighting, and by mild exercise on the treadmill. In all these conditions, stroke volume attains values larger than those measured during recumbent rest. Though we have never subjected our cats to heavy or very prolonged exertional tasks, it seems likely that in these conditions stroke volume might increase even further. Indeed, this trend is apparent in Fig. 6, where we have 
compared short (less than $10 \mathrm{sec}$ ) with long (20 or $30 \mathrm{sec}$ ) fighting trials : in long fighting the heart rate did not increase beyond the peaks attained in shorter episodes and the progressive increase in cardiac output with protraction of fighting was almost entirely obtained by a continuing increase in stroke volume.

It appears, therefore, that an increase in stroke volume is a mechanism ordinarily called upon in the cat when natural behaviour requires an increase in cardiac output, while in man it is called upon in extraordinary conditions only, during strenuous exercise or when the heart is artificially set at high basal levels by atrial pacing or cholinergic blockade. Even in these conditions, as in the experiments by Ross et al. (1965) and by Bevegard (1963), the mean stroke volume increase during exercise is not above supine resting values recorded when the heart rate was spontaneously low. Furthermore, the observation that in the cat stroke volume can increase above resting recumbent levels upon the simple assumption of the standing posture, when gravitational effects should cause, if anything, a decrease in venous return, also indicates that at least in these conditions the stroke volume increase does not depend on a primary increase in venous return.

The fact that a stroke volume regulating mechanism is ordinarily operative in the cat shows that it has a physiological significance, and supports the idea that also in man exertion is accompanied by stimulation of myocardial contractility. The emphasizing or overshadowing of this inotropic effect in cat or man respectively would depend on the different autonomic bal. ance regulating the heart rate in the two species. Stimulation of myocardial contractility can be translated into an actual increase in stroke volume only when vagal tone is already very low in base-line conditions, and its suppression does not cause great rises in heart rate. This condition occurs naturally in the cat. In man, suppression of the high vagal tone even during mild exertion makes the heart rate increase remarkably, and thus generally conceals the myocardial augmentative effect. Our results on the cat support previous indications in physiological literature (see: Sarnoff and Mitchell, 1962) and more precisely suggest that the concealed inotropic effect during exertion in man might be measured from the difference between the maintained stroke volume in spite of an increased heart rate during exertion, and the linear inverse relationship between heart rate and stroke volume calculated by Ross et al. (1965) during atrial pacing at increased rates. A myocardial agmentative effect is of course known to occur during exercise in pathological conditions, such as complete heart block with fixed slow rate (Bevegärd, 1962; Benchimol and Dimond, 1964; Segel et al., 1964), but what we have tried to emphasize here is that it can represent a physiological mechanism operative, though masked, in the circulatory adjustment of normal subjects.

Though it seems reasonable that the limited ability of the cat to increase its heart rate cluring exertion may be the main factor making stroke volume regulation so very evident in this species, it might be argued that an equally important factor may be the much more limited gravitational effects of standing upon venous return in a small quadruped like the cat, compared to head-up standing in man. Though the small hydrostatic gradient of cats may undoubtedly be a factor, it does not seem to be the crucial one, however. Indeed the dog, in which gravitational effects are certainly closer to those operative in the cat than to those present in man, but whose heart rate regulation is dominated by a strong resting vagal tone as in man, behaves much the same as man in terms of stroke volume regulation: stroke volume is decreased during sitting or standing (even active standing with a well maintained venous return: Rushmer, 1959; Abel and Waldhausen, 1968), and during exercise cardiac output is increased exclusively through tachycardia with practically no change in stroke volume (Wang et al., 1960 a). A further similarity between the stroke volume response of dog and man during exercise occurs when the usual heart rate increase is artificially prevented or reduced: in these conditions the dog's cardiac output is mainly or exclusively augmented through a rise in stroke volume (Wang et al., 1960a; Warner and Toronto, 1960), much the same as occurs in man (Ross et al., 1965).

Some comments can finally be made on the vasomotor changes we have recorded during an active change of posture from lying to standing. They are dominated by vasodilatation in active hindlimbs, only partially compensated by visceral vasoconstriction, as exemplified by a fall in mesenteric conductance in our records. Hence there is an increase in total peripheral conductance and generally some decrease in blood pressure, indicating predominance of metabolic vasodilatation over neural vasoconstriction. On the whole the vasomotor adjustments during active changes in posture closely resemble those occuring during mild exercise on the treadmill (Adams et al., 1970) and confirm that, at least in the cat, exertional predominate over gravitational factors in regulating circulation during active standing. Under this aspect, our observations further caution against indiscriminately applying to active changes in posture data obtained during passive tilting.

\section{References}

Abel, F. L., Waldhausen, J. A.: Influence of posture and passive tilting on venous return and cardiac output. Amer. J. Physiol. 215, 1058 (1968).

Adams, D. B., Baccelli, G., Mancia, G., Zanchetti, A.: Cartiovascular changes during naturally elicited fighting behavior in the cat. Amer. J. Physiol. 216, 1266 (1969).

- - - Relation of cardiovascular changes in fighting to emotion and exercise. J. Physiol. (Lond.) 1970 (in press).

Benchimol, A., Li, Y. B., Dimond, E. G.: Cardiovascular dynamics in complete heart block at various heart rates. Effect of exercise at a fixed heart rate. Circulation $\mathbf{3 0}, \mathbf{5 4 2}$ (1964).

Beveguird, S.: Observations on the effect of varying ventricular rate on the circulation at rest and during exercise in two patients with an artificial pacemaker. Acta med. scand. $172,615(1962)$. 
Bevegåd, S.: The effect of cardioacceleration by methylscopolamine-nitrate on the circulation at rest and during exercise in supine position, with special reference to the influence on the stroke volume. Acta physiol. scand. 57, 61 (1963).

- Holmgren, A., Jonsson, B.: The effect of body position on the circulation at rest and during exercise, with special reference to the influence on stroke volume. Acta physiol. scand. 49, 279 (1960).

- Shepherd, J. T.: Regulation of the circulation during exercise in man. Physiol. Rev. 47, 178 (1967).

Bristow, J. D., Honour, A. J., Pickering, T. G., Sleight, P.: Cardiovascular and respiratory changes during sleep in normal and hypertensive subjects. Cardiovasc. Res. $\mathbf{3}, \mathbf{4 7 6}$ (1969).

Chapman, C. B., Fisher, J. N., Sproule, B. J.: Behavior of stroke volume at rest and during exercise in human beings. J. clin. Invest. 39, 1208 (1960).

Damato, A. N., Galante, J. G., Smith, W. M.: Hemodynamic response to treadmill exercise in normal subjects. J. appl. Physiol. 21, 959 (1966).

Guazzi, M., Mancia, G., Kumazawa, T., Baccelli, G., Zanchetti, A.: Effects of cardiac denervation on blood pressure and heart rate during natural sleep in the cat. Cardiovasc. Res. 2,265 (1968).

- Zanchetti, A.: Blood pressure and heart rate during natural sleep of the cat and their regulation by carotid sinus and aortic reflexes. Arch. ital. Biol. 108, 789 (1965).

Robinson, B. F., Epstein, S. E., Beiser, G. D., Braunwald, E.: Control of heart rate by the autonomic nervous system. Studies in man on the interrelation between baroreceptor mechanisms and exercise. Circulat. Res. 19, 400 (1966).
Ross, J., Jr., Linhart, J. W., Braunwald, E. ; Effects of chan. ging heart rate in man by electrical stimulation of the right heart. Studies at rest, during exercise, and with isoproterenol. Circulation 39, 549 (1965).

Rushmer, R. F.: Postural effects on the baseline of ventricular performance. Circulation 90, 897 (1959).

Sarnoff, S. J., Mitchell, J. H.: The control of the function of the heart. In: Handbook of physiology, sec. 2, Circulation (W. F. Hamilton and P. Dow, eds.), vol. 2, p. 489. Washington, D. C.: American Physiological Society 1962.

Segel, N., Hudson, W. A., Harris, P., Bishop, J. M.: The circulatory effects of electrically induced changes in ventricular rate at rest and during exercise in complete heart block. J. clin. Invest. 43, 1541 (1964).

Snedecor, G. W., Cochran, W. G.: Statistical methods, 6 th ed. Ames, Iowa: The Iowa State University Press 1967.

Stark, R. D.: Conductance or resistance ? Nature (Lond.) 117, 799 (1968).

Wang, Y., Marshall, R. J., Shepherd, J. T.: Stroke volume in the dog during graded exercise. Circulat. Res. 8, 558 (1960a).

- - The effect of changes in posture and of graded exercise on stroke volume in man. J. clin. Invest. 39, 105] (1960b).

Warner, H. R., Toronto, A. F.: Regulation of cardiac output through stroke volume. Circulat. Res. 8, 549 (1960).

Prof. Dr. A. Zanchetti

Istituto di Ricerche Cardiovascolari dell' Università di Milano

Via F. Sforza, 35

I-20122 Milano 